\section{IAP Immunization Timetable 2012: Some Issues}

This refers to the Consensus Recommendations and IAP Immunization Timtable 2012 [1]; the issues of pertussis vaccine for adolescents and young adults and second dose of varicella vaccine have not been addressed. IAP should come out with some indigeneous vaccine for protection of pertussis in adolescents and adults.

IAP also needs to deliberate on two other issues: $(i)$ why do we need 2 nd dose of varicella vaccine to prevent breakthrough disease with low vaccine coverage? In the USA need for second dose arose when vaccine coverage increased, resulting in low boosting effect provided by subclinical infections. Many cases of breakthrough disease have been seen recently. Could it be a case of poor response to varicella vaccine as has been observed that some Indian children had shown poor response to OPV? and (ii) what should be the interval between two doses of vaccine to prevent breakthrough disease in Indian children?

Yash Paul A-D-7, Devi Marg, Bani Park, Jaipur-302016, India. dryashpaul2003@yahoo.com

\section{REFERENCE}

1. Consensus Recommendations on Immunization and IAP Immunization Timetable 2012. Indian Pediatr. 2012;49:549-64.

\section{REPLY}

It is not feasible to discuss all the issues related to all the available vaccines in a single meeting. Regarding your suggestion on use of reduced quantity of whole cell pertussis vaccines to adolescents and adults (through DTP/DT/Td), we cannot recommend this until adequate trials are conducted with proper protocol and after proper approval and clearance from national regulatory authority (NRA). Moreover, it is beyond the purview of IAPCOI to produce an indigenous affordable Tdap/ pertussis vaccine for the adolescents.

IAPCOI has recommended two dose schedule for prevention of 'breakthrough varicella' [1]. Following are the rationale behind IAPCOI's recommendations:

1. The recommended ages for routine first (at age 12-15 months) and second (at age 4-6 years) doses of varicella vaccine are harmonized with the recommendations for MMR vaccine use and intended to limit the period when children have no varicella antibody. The recommended age for the second dose is supported by the current epidemiology of varicella, with low incidence and few outbreaks among preschool-aged children and higher incidence and more outbreaks among elementary school-aged children.

2. Although, most studies are done when 2 nd dose is given after 3 months of the first, there are few trials where the two schedules were compared and it was concluded that among children, VZV antibody levels and GMTs after 2 doses administered 4-6 years apart were comparable to those obtained when the 2 doses were administered 3 months apart (seroconversion: 99.2\% vs 99.6\%, GMTs: 212.4 vs 142.6, respectively).

3. However, the CMI responses measured by mean stimulation index (SI), a marker of cell-mediated immunity were 36.9 for 2 nd dose after 3 months of primary dose, and 58.6 when 2 nd dose was given at 46 yrs of age [2].

VIPIN M VASHISHTHA
Convener, IAP Committee on Immunization,
Mangla Hospital\& Research Center,
Shakti Chowk,
Bijnor,Uttar Pradesh, 246701,India
$v m v @ m a n g l a h o s p i t a l . o r g$

\section{REFERENCES}

1. IAP Guidebook on Immunization, 2009-11. Eds. Yewale $\mathrm{V}$, Choudhury P, Thacker N. Individual vaccines; Varicella vaccine. Available from: http://www.iapcoi. $\mathrm{com} / \mathrm{hp} / \mathrm{pdf} / \mathrm{IAP} \% 20 \mathrm{GUIDE} \% 20 \mathrm{BOOK} \% 20 \mathrm{ON} \% 20$ IMMUNIZATION\%20INDIVIDUAL\%20VACCINES.pdf. Accessed on September 4, 2012.

2. MMWR, Recommendations and Reports. Prevention of Varicella. Recommendations of the Advisory Committee on Immunization Practices (ACIP). June 22, 2007/ 56(RR04);1-40. Available from: http://www.cdc.gov/ $\mathrm{mmwr} /$ preview/mmwrhtml/rr5604a1.htm. 